\title{
Free Vibration Analysis of Functionally Heterogeneous Hollow Cylinder Based on Three Dimensional Elasticity Theory
}

\author{
Masoud Asgari \\ Faculty of Mechanical Engineering, K. N. Toosi University of Technology, Pardis Street, Molla-Sadra Avenue, \\ Vanak Square, Tehran, Iran
}

\begin{abstract}
(Received 16 November 2014; accepted 13 January 2016)
A two-dimensional functionally heterogeneous thick hollow cylinder with a finite length is considered and its natural modes and frequencies are determined. Since mode shapes of a thick cylinder are three-dimensional, even with axisymmetric conditions, three-dimensional theory of elasticity implemented for problem formulation. The axisymmetric conditions are assumed for the cylinder. The material properties of the two-dimensional functionally graded material (2D-FGM) cylinder are varied in the radial and axial directions with power law functions. Effects of volume fraction distribution on the different types of anti-symmetric mode shape configuration and vibrational behaviour of a simply supported cylinder are analyzed. Three-dimensional equations of motion are used and the eigen value problem is developed based on direct variational method. The study shows that the 2D-FGM cylinder exhibit interesting vibrational characteristics and mode shapes when the constituent volume fractions are varied.
\end{abstract}

\section{INTRODUCTION}

Recently, the composition of several different materials is often used in structural components in order to optimize the responses of structures subjected to severe loadings. For reducing the local stress concentrations induced by abrupt transitions in material properties, the transition between different materials is made gradually. This idea leads to the concept of functionally graded materials (FGMs). ${ }^{1}$ The mechanical properties of FGMs vary continuously between several different materials. Most research in this area is concerned with thermoelastic and residual stress analysis. In many applications of these materials the vibrational characteristics are of great importance in addition to stress considerations. The vibration of FGM cylindrical structures has been studied by a number of researchers..$^{2-7}$ Different studies on the vibration of cylindrical shells made of a FGM based on Love's and some other classical shell theories have been done and usually Rayleigh-Ritz and finite element methods used for solving governing equations. $^{2-7}$

While the vibrational behaviour of a thick walled cylinder is of considerable engineering importance, all of the previously discussed papers are mainly focused on cylindrical shells using the classical equations of thin shell theories, except Chen et al. ${ }^{7}$ who investigated a thick hollow cylinder using equations of piezoelasticity based on laminate model as opposed to classical shell theories. Classical or thin-shell theories are based on the simplifying assumptions of Kirchhof-Love's hypothesis. This omission makes the thin-shell theories highly inadequate for the analysis of even slightly thick shells. ${ }^{8}$ The higher order shell theories are better than the thin-shell theories for the analysis of slightly thick shells but are still inadequate for the analysis of moderately thick shells. To analyze moderately thick shells, the transverse normal stress and strain components, which are ignored in the higher ordered shell theories, have to be accounted for and only an analysis based on the three-dimensional theory of elasticity would account for all the transverse stress and strain components. ${ }^{8}$
Few studies have been conducted for thick hollow circular cylinders. They require a three-dimensional analysis, which is based on the theory of elasticity. As a result, in the literatures, the study of free vibrations of thick circular cylinders using three-dimensional theory of elasticity is relatively scarce in comparison to the study of thin shells using other shell theories. Studies on shells based on three-dimensional theory of elasticity have been presented by some researchers for infinitely long cylindrical shells. ${ }^{9-12}$ For finite-length thick cylindrical shells, different methods such as the finite element method, series solution, and the Ritz energy method have been used by some researchers for both solid and hollow homogeneous cases. ${ }^{13-17}$ A three-dimensional energy formulation was used by Liew et. al. ${ }^{18}$ to compute frequencies and develop graphical representation for three-dimensional mode shapes of a homogeneous hollow cylinder. Loy and Lam ${ }^{8}$ also presented an approximate analysis by using a layerwise approach to study the vibration of thick circular cylindrical shells on the basis of three-dimensional theory of elasticity. Buchanan and Yii $^{19}$ investigated the effect of symmetrical boundary conditions on the vibration of thick hollow cylinders using finite element method. Other researchers ${ }^{20-23}$ have also presented studies using three-dimensional theory of elasticity based on the Rayleigh-Ritz method for homogeneous and laminated cylinders.

Most of the referred studies considered ways of determining the frequencies of cylinders. However, very few of the authors give a description of the mode shapes of the thick cylinders. While mode shapes are also very important sources of information for understanding and controlling the vibration of a structure. ${ }^{26}$ Singal and Williams ${ }^{24}$ combined experimental results with a Ritz energy method of analysis to compare frequencies for free-free cylinders. They gave a description for the mode shapes of thick- walled hollow cylinders and rings. Wang and Williams ${ }^{25}$ have studied extensively frequencies and mode shapes of finite length hollow cylinder using a commercial finite element code and three-dimensional block elements for their analysis. Singhal et al. ${ }^{26}$ presented theoretical and 
experimental modal analysis by using a thick-walled circular cylinder model to obtain its natural frequencies and mode shapes.

On the other hand, in the previous discussed literature, vibration analysis of moderately thick- walled hollow cylinders are limited to isotropic and laminated cylinders and functionally graded thick hollow cylinders with finite length were not seen in the literatures. Also the functionally graded cylindrical shells considered using thin shell theories. So, investigation of a functionally graded thick hollow cylinder can be of great importance. Additionally, in all of the discussed cases, the variation of volume fraction and properties of the FGMs are one-dimensional and the properties vary continuously from the inner surface to the outer one with a prescribed function. But a conventional functionally graded material may also not be so effective in some design problems since all outer or inner surfaces will have the same composition distribution while in advanced machine elements, load distribution may change in two or three directions. ${ }^{27}$ Therefore, if the FGM has two-dimensional dependent material properties more effective material resistance can be obtained. Based on this fact, a two-dimensional FGM whose material properties are bi-directionally dependent is introduced. Recently a few authors have investigated 2D-FGM especially its stress analysis. $^{27-36}$

The author also has investigated the natural frequencies of a thick hollow cylinder with finite length made of 2D-FGM. ${ }^{37}$ The influence of constituent volume fractions on natural frequencies was studied by varying the volume fractions of the constituent metals and ceramics. Furthermore, the effects of length and thickness of the cylinder on fundamental natural frequency were considered in different types of 2D-FGM cylinder.

So far, investigation of mode shapes configuration of FGM thick finite cylinder has not been considered in previous studies. Hence, in order to extend the previous studies and to investigate the mode shape configuration of an FGM thick hollow cylinder, the free vibration analysis and all types of mode shapes of a thick hollow cylinder with finite length made of 2D-FGM on the basis of three- dimensional theory of elasticity has been considered in this study. The material properties of the cylinder are varied in the radial and axial directions with power law functions. Effects of volume fraction distribution and FGM configuration on the natural frequencies and three-dimensional mode shapes of a simply supported functionally graded thick hollow cylinder are analyzed. The influence of constituent volume fractions is studied by varying the volume fractions of the constituent metals and ceramics. A functionally graded cylinder with twodimensional gradation of distribution profile has been investigated as well as the one-dimensional gradation of material distribution. Three-dimensional equations of motion are used and the eigen value problem is developed based on Rayleigh-Ritz variational method and all non- axisymmetric mode shapes are considered. The finite element method with graded material characteristics within each element of the structure is used for the solution. Using conventional finite element formulations such that the property field is constant within an individual element for dynamic problems leads to significant discontinuities and inaccuracies. ${ }^{38}$ These inaccuracies will be more significant in 2D-FGM cases. On the other hand, by using graded finite element in which the material property field is graded continu-

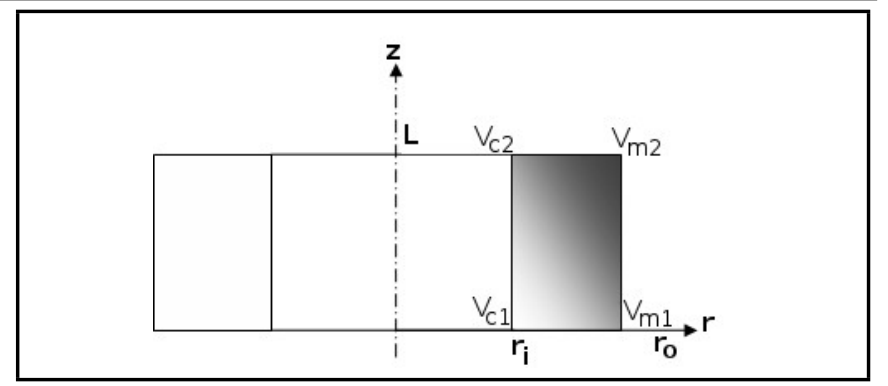

Figure 1. Axisymmetric cylinder with two dimensional material distributions. $^{37}$

ously through the elements, accuracy can be improved without refining the mesh size. ${ }^{39,40}$ Based on these facts the graded finite element developed by the author ${ }^{41}$ is used for modeling of the present problem.

\section{PROBLEM FORMULATION}

In this section, the volume fraction distributions in the two radial and axial directions are introduced. The 3D governing equations of motion in cylindrical coordinates are obtained and the graded finite element is used for modeling the nonhomogeneity of the material.

\subsection{Volume Fraction and Material Distribution in 2D-FGM Cylinder}

In the conventional one dimensional functionally graded cylinder, the cylinder's material is graded through the radial direction. The cylinder is made of a combined metal-ceramic material for which the mixing ratio is varied continuously in the $r$-direction from pure ceramic in the inner surface to pure metal in the outer surface, or vice versa. In such cases, the volume fraction variation of the metal is proposed as a power law relation. ${ }^{37}$ Using the rule of mixtures, the distribution of material properties is obtained.

Significant advances in fabrication and processing techniques have made it possible to produce FGMs using processes that allow FGMs with complex properties and shapes, including two-and three- dimensional gradients using computeraided manufacturing techniques. 2D-FGMs are usually made by continuous gradation of three or four distinct material phases that one or two of them are ceramics and the others are metal alloy phases. The volume fractions of the constituents vary in a predetermined composition profile. Now consider the volume fractions of 2D-FGM at any arbitrary point in the axisymmetric cylinder of internal radius $r_{i}$, external radius $r_{o}$, and finite length $L$ shown in Fig. 1 In the present cylinder the inner surface is made of two distinct ceramics and the outer surface from two metals. The variables $c 1, c 2, m 1$, and $m 2$ denote first ceramic, second ceramic, first metal and second metal, respectively.

The volume fraction of the first ceramic material is changed from $100 \%$ at the lower surface to zero at the upper surface by a power law function. Additionally, this volume fraction is changed continuously from inner surface to the outer surface. The volume fractions of the other materials change similar to the mentioned one in two directions. The function of volume fraction distribution of each material can be explained as ${ }^{37}$

$$
V_{c 1}(r, z)=\left[1-\left(\frac{r-r_{i}}{r_{0}-r_{i}}\right)^{n_{r}}\right]\left[1-\left(\frac{z}{L}\right)^{n_{z}}\right]
$$


Table 1. Basic constituents of the 2D-FGM cylinder.

\begin{tabular}{||c|c|c|c|}
\hline Constituents & Material & $E(\mathrm{Gpa})$ & $\rho\left(\mathrm{kg} / \mathrm{m}^{3}\right)$ \\
\hline$m 1$ & Ti6Al4V & 115 & 2715 \\
\hline$m 2$ & $\mathrm{~A} 11100$ & 69 & 4515 \\
\hline$c 1$ & $\mathrm{SiC}$ & 440 & 3210 \\
\hline$c 2$ & $\mathrm{Al}_{2} \mathrm{O}_{3}$ & 150 & 3470 \\
\hline
\end{tabular}

$$
\begin{gathered}
V_{c 2}(r, z)=\left[1-\left(\frac{r-r_{i}}{r_{0}-r_{i}}\right)^{n_{r}}\right]\left[\left(\frac{z}{L}\right)^{n_{z}}\right] \\
V_{m 1}(r, z)=\left(\frac{r-r_{i}}{r_{0}-r_{i}}\right)^{n_{r}}\left[1-\left(\frac{z}{L}\right)^{n_{z}}\right] ; \\
V_{m 2}(r, z)=\left(\frac{r-r_{i}}{r_{0}-r_{i}}\right)^{n_{r}}\left(\frac{z}{L}\right)^{n_{z}} ;
\end{gathered}
$$

where $n_{r}$ and $n_{z}$ are non-zero parameters that represent the basic constituent distributions in $r$ and $z$ directions. Material properties at each point can be obtained by using the linear rule of mixtures, in which a material property $P$ at any arbitrary point $(r, z)$ in the 2D-FGM cylinder is determined by linear combination of volume fractions and material properties of the basic materials as ${ }^{37}$

$$
P=P_{c 1} V_{c 1}+P_{c 2} V_{c 2}+P_{m 1} V_{m 1}+P_{m 2} V_{m 2}
$$

The basic constituents of the 2D-FGM cylinder are presented in Table 1. It should be noted that Poisson's ratio is assumed to be constant through the body. This assumption is reasonable because of the small differences between the Poisson's ratios of basic materials.

\subsection{Governing Equations}

Consider a 2D-FGM thick hollow cylinder of internal radius, $r_{i}$ external radius $r_{o}$, and finite length $L$. Because of the axisymmetry of geometry and material distribution profile, coordinates $r, z$, and $\theta$ are used in the analysis. Neglecting body forces, the equations of motion in cylindrical coordinates are obtained as

$$
\begin{gathered}
\frac{\partial \sigma_{r r}}{\partial r}+\frac{\partial \tau_{r z}}{\partial z}+\frac{\partial \tau_{r \theta}}{r \partial \theta}+\frac{\sigma_{r r}-\sigma_{\theta \theta}}{r}=\rho(r, z) \frac{\partial^{2} u}{\partial t^{2}} \\
\frac{\partial \tau_{r \theta}}{\partial r}+\frac{\partial \sigma_{\theta \theta}}{r \partial \theta}+\frac{\partial \tau_{z \theta}}{\partial z}+\frac{2 \tau_{r \theta}}{r}=\rho(r, z) \frac{\partial^{2} v}{\partial t^{2}} \\
\frac{\partial \tau_{r z}}{\partial r}+\frac{\partial \tau_{z \theta}}{r \partial \theta}+\frac{\partial \sigma_{z z}}{\partial z}+\frac{\tau_{r z}}{r}=\rho(r, z) \frac{\partial^{2} w}{\partial t^{2}}
\end{gathered}
$$

where $u, v$, and $w$ are radial, circumferential, and axial components of displacement respectively those are functions of $(r, z, \theta, t)$ and $\rho(r, z)$ is the mass density that depends on $r$ and $z$ coordinates. The constitutive equations for FGM are written as

$$
\left\{\sigma_{i j}\right\}=[D(r, z)]\left\{\varepsilon_{i j}\right\}
$$

where the stress and strain components and the coefficients of elasticity are

$$
\begin{aligned}
& \left\{\sigma_{i j}\right\}=\left[\begin{array}{llllll}
\sigma_{r r} & \sigma_{\theta \theta} & \sigma_{z z} & \tau_{z \theta} & \tau_{r z} & \tau_{r \theta}
\end{array}\right] ; \\
& \left\{\varepsilon_{i j}\right\}=\left[\begin{array}{llllll}
\varepsilon_{r r} & \varepsilon_{\theta \theta} & \varepsilon_{z z} & \gamma_{z \theta} & \gamma_{r z} & \gamma_{r \theta}
\end{array}\right] ;
\end{aligned}
$$

$$
\begin{aligned}
& {[D(r, z)]=\frac{E(r, z)}{(1+\nu)(1-2 \nu)}} \\
& {\left[\begin{array}{cccccc}
1-\nu & \nu & \nu & 0 & 0 & 0 \\
\nu & 1-\nu & \nu & 0 & 0 & 0 \\
\nu & \nu & 1-\nu & 0 & 0 & 0 \\
0 & 0 & 0 & \frac{1-2 \nu}{2} & 0 & 0 \\
0 & 0 & 0 & 0 & \frac{1-2 \nu}{2} & 0 \\
0 & 0 & 0 & 0 & 0 & \frac{1-2 \nu}{2}
\end{array}\right]}
\end{aligned}
$$

where $\nu$ denotes the Poison's ratio, which is uniform through the cylinder and $E(r, z)$ is Young's modulus that depends on $r$ and $z$ coordinates. The strain-displacement equations are: ${ }^{42}$

$$
\begin{gathered}
\varepsilon_{r}=\frac{\partial u}{\partial r} \\
\varepsilon_{\theta}=\frac{\partial v}{r \partial \theta}+\frac{u}{r} \\
\varepsilon_{z}=\frac{\partial w}{\partial z} \\
\gamma_{r z}=\frac{\partial u}{\partial z}+\frac{\partial w}{\partial r} \\
\gamma_{z \theta}=\frac{\partial v}{\partial z}+\frac{\partial w}{r \partial \theta} \\
\gamma_{r \theta}=\frac{\partial u}{r \partial \theta}+\frac{\partial v}{\partial r}-\frac{v}{r} .
\end{gathered}
$$

The cylinder is simply supported on its two end edges. So mechanical boundary conditions on upper and lower edges are assumed as

$v(r, 0, \theta, t)=v(r, L, \theta, t)=w(r, 0, \theta, t)=w(r, L, \theta, t)=0$.

A solution that satisfies the circumferential displacement and defines a circular frequency is ${ }^{19}$

$$
\begin{aligned}
& u(r, \theta, z, t)=\psi_{1}(r, z) \cos (m \theta) e^{i \omega t} ; \\
& v(r, \theta, z, t)=\psi_{2}(r, z) \sin (m \theta) e^{i \omega t} ; \\
& w(r, \theta, z, t)=\psi_{3}(r, z) \cos (m \theta) e^{i \omega t} ;
\end{aligned}
$$

where $m$ is the circumferential wave number and $\omega$ is the circular frequency. Also considering the circumferential symmetry of the cylinder about the coordinate $\theta$, the displacement amplitude functions can be written as $\psi_{1}(r, z), \psi_{2}(r, z)$ and $\psi_{3}(r, z)$. It is obvious that $m=0$, which donates the axisymmetric vibration. Certain specified uniform boundary conditions along the two ends can be satisfied by choosing the displacement amplitude functions properly.

In order to solve the governing equations the finite element method with graded element properties is used. For this purpose, the variational formulation is considered. In conventional finite element formulations a predetermined set of material properties are used for each element such that the property field is constant within an individual element. For modeling a continuously non-homogeneous material, the material property function must be discretized according to the size of elements mesh. This approximation can provide significant discontinuities. In addition, variation of material properties in 
two directions such as the present problem makes this effect more considerable. Based on these facts the graded finite element is strongly preferable for modeling of the present problem. Hamilton's principle for the present problem is

$$
\int_{t 1}^{t 2} \delta(\Pi-T) d t=0 ;
$$

where $\Pi$ and $T$ are potential energy and kinetic energy respectively. These functions and their variations are

$$
\begin{gathered}
T=\frac{1}{2} \iiint_{\text {vol }} \rho(r, z)\left(\left(\frac{\partial u}{\partial t}\right)^{2}+\left(\frac{\partial v}{\partial t}\right)^{2}+\left(\frac{\partial w}{\partial t}\right)^{2}\right) d V \\
\delta T=\iiint_{\text {vol }} \rho(r, z)\left(\left(\frac{\partial^{2} u}{\partial t^{2}}\right) \delta u+\left(\frac{\partial^{2} v}{\partial t^{2}}\right) \delta v+\right. \\
\left.+\left(\frac{\partial^{2} w}{\partial t^{2}}\right) \partial w\right) d V \\
\Pi=\frac{1}{2} \iint_{\text {vol }}\left\{\sigma_{i j}\right\}^{T}\left\{\varepsilon_{i j}\right\} d V \\
\delta \Pi=\frac{1}{2} \iiint_{\text {vol }}\left\{\sigma_{i j}\right\}^{T}\left\{\delta \varepsilon_{i j}\right\} d V
\end{gathered}
$$

where $V$ denotes the area and volume of the domain under consideration. Substituting Eqs. (12) to (15) in Hamilton's principle and applying side conditions, $\delta u\left(t_{1}\right)=\delta u\left(t_{2}\right)=\delta v\left(t_{1}\right)=$ $\delta v\left(t_{2}\right)=\delta w\left(t_{1}\right)=\delta w\left(t_{2}\right)=0$, by part integration have

$$
\begin{array}{r}
\iiint_{\text {vol }}\left\{\sigma_{i j}\right\}^{T}\left\{\delta \varepsilon_{i j}\right\} d V+\iiint_{\text {vol }} \rho(r, z)\left(\left(\frac{\partial^{2} u}{\partial t^{2}}\right) \delta u+\right. \\
\left.+\left(\frac{\partial^{2} v}{\partial t^{2}}\right) \delta v+\left(\frac{\partial^{2} w}{\partial t^{2}}\right) \partial w\right) d V=0
\end{array}
$$

The strain-displacement relations can be written as ${ }^{43}$

$$
\{\varepsilon\}=[L]\{u\}
$$

where $[L]$ relates displacements into strain components. And

$$
\{u\}=\left(\begin{array}{c}
u \\
v \\
w
\end{array}\right) .
$$

Four node tetrahedral element is used to discrete the domain. By taking the nodal values of $u, v$, and $w$ as the degrees of freedom a linear displacement model can be assumed as

$$
\left(\begin{array}{c}
u \\
v \\
w
\end{array}\right)^{e}=[N]\left\{Q_{0}^{e}\right\} e^{i \omega t}
$$

where $[N]$ is the matrix of assumed shape functions and satisfy certain specified boundary conditions and $\left\{Q_{0}^{e}\right\}$ is the nodal displacement vector of element. The matrix of interpolation functions corresponding to elements which derived in terms of global coordinates of nodes of elements its components are three-dimensional and axisymmetric. Vector of nodal displacements (degrees of freedom) is

$\left\{Q^{e}\right\}=$ $\left\{\begin{array}{llllllllllll}u_{i} & v_{i} & w_{i} & u_{j} & v_{j} & w_{j} & u_{k} & v_{k} & w_{k} & u_{l} & v_{l} & w_{l}\end{array}\right\}^{T} ;$

where subscripts $i, j, k, l$ are related to four nodes of each element.

The cylinder will be divided into some brick-like subdivisions in radial, axial, and circumferential directions as well as making a tetrahedral mesh through the use of brick subdivisions. In this case $10 * 10 * 12$ brick subdivisions produced that leads to 2400 elements including 4356 degrees of freedom. Applying Hamilton's principle for each element, it can be achieved as

$$
\begin{array}{r}
\delta\left\{Q^{e}\right\}^{T}\left[\iint_{V^{e}}[B(r, z, \theta)]^{T}[D(r, z)][B(r, z, \theta)] d V\right]\left\{Q^{e}\right\}+ \\
+\delta\left\{Q^{e}\right\}^{T}\left[\iiint_{V^{e}} \rho(r, z)[N]^{T}[N] d V\right]\left\{\ddot{Q}^{e}\right\}=0
\end{array}
$$

where $V$ is the volume of element and $[B]$ is the operation matrix of strain-nodal displacement. In graded finite element, the interpolation function for the displacements within the elements and strain-displacement relations are the same as standard conventional finite. In this way the constitutive relation is

$$
\left\{\sigma_{i j}\right\}=[D(r, z)]\left\{\varepsilon_{i j}\right\}
$$

where the components of $[D(r, z)]$ could be explicit functions describing the actual material property gradient in which $E(r, z)$ is determined at each point through the element using distribution function of this property based on rule of mixtures as

$$
\begin{array}{r}
E(r, z)=E_{c 1} V_{c 1}(r, z)+E_{c 2} V_{c 2}(r, z)+ \\
+E_{m 1} V_{m 1}(r, z)+E_{m 2} V_{m 2}(r, z) .
\end{array}
$$

Also, the mass density $\rho(r, z)$ is, in general, a function of position as well as the mechanical properties. Therefore, in the graded finite element the mass density distribution should be assigned into the element formulation as

$$
\begin{gathered}
\rho(r, z)=\rho_{c 1} V_{c 1}(r, z)+\rho_{c 2} V_{c 2}(r, z)+ \\
+\rho_{m 1} V_{m 1}(r, z)+\rho_{m 2} V_{m 2}(r, z) .
\end{gathered}
$$

Since $\delta\left\{Q^{e}\right\}$ is the variational displacement of the nodal points and is arbitrary, it can be omitted from Eq. (23), and then this equation can be written as

$$
[M]^{e}\left\{\ddot{Q}^{e}\right\}+[K]^{e}\left\{Q^{e}\right\}=0
$$

where the characteristic matrices are given as

$$
\begin{aligned}
{[K]^{e} } & =\iiint_{V^{e}}[B(r, z, \theta)]^{T}[D(r, z)][B(r, z, \theta)] d V ; \\
{[M]^{e} } & =\iiint_{V e}[N(r, z, \theta)]^{T}[N(r, z, \theta)] \rho(r, z) d V .
\end{aligned}
$$


Table 2. Dimensionless frequencies $\phi=(\omega H / \pi) \sqrt{\rho / G}$ of axisymmetric vibration for freely supported isotropic cylinders $(m=0, H / R=0.4, \nu=0.3)$.

\begin{tabular}{|c|c|c|c|c|c|c|c|}
\hline \hline$H / L$ & Method & $\phi_{1}$ & $\phi_{2}$ & $\phi_{3}$ & $\phi_{4}$ & $\phi_{5}$ & $\phi_{6}$ \\
\hline \multirow{3}{*}{0.2} & (Armenakas et al., 1969) & 0.20495 & 0.34765 & 1.07205 & 1.82336 & 2.09588 & 3.00073 \\
\cline { 2 - 8 } & (Cheung and Wu, 1972) & 0.20495 & 0.34765 & 1.07312 & 1.82688 & 2.10257 & 3.02850 \\
\cline { 2 - 8 } & Present method & 0.20492 & 0.34763 & 1.07309 & 1.82686 & 2.10254 & 3.02848 \\
\hline \multirow{3}{*}{0.4} & (Armenakas et al., 1969) & 0.27540 & 0.67185 & 1.23591 & 1.76178 & 2.25224 & 2.99980 \\
\cline { 2 - 8 } & (Cheung and Wu, 1972) & 0.27544 & 0.67188 & 1.23712 & 1.76587 & 2.25874 & 3.02742 \\
\cline { 2 - 8 } & Present method & 0.27539 & 0.67182 & 1.23704 & 1.76580 & 2.25868 & 3.02735 \\
\hline \multirow{3}{*}{0.6} & (Armenakas et al., 1969) & 0.42022 & 0.98133 & 1.44860 & 1.75005 & 2.43159 & 3.00691 \\
\cline { 2 - 8 } & (Cheung and Wu, 1972) & 0.42038 & 0.98145 & 1.45008 & 1.75433 & 2.43851 & 3.03437 \\
\cline { 2 - 8 } & Present method & 0.42032 & 0.98138 & 1.45001 & 1.75421 & 2.43842 & 3.03428 \\
\hline
\end{tabular}

For finding the components of characteristic matrices, the integral must be taken over the elements' volume considering Eqs. (23) and (24). As $[D(r, z)]$ and $\rho(r, z)$ are not constant, these matrices are evaluated by numerical integration for each element.

Now by assembling the element matrices, the global matrix equation for the structure can be obtained as

$$
[M]\{\ddot{Q}\}+[K]\{Q\}=\{0\} .
$$

Once the finite element equations are established, $Q=$ $Q_{0} e^{i \omega t}$ was substituted into Eq. (28) which resulted in an eigen value equation that can be solved using standard eigen value extraction procedures. To get a better illustration of the mode shapes, the numerical results of the displacements relate to each eigen value on each node were transferred into the proper coordinate system and the radial, tangential, and longitudinal directions of the nodes determined.

\section{NUMERICAL RESULTS AND DISCUSSION}

To verify the present solution, as similar works to the present work are few, a finite length of a homogeneous thick cylinder that can be found with the existing literature is used. A finite element for axisymmetric elasticity is formulated directly in the cylindrical coordinates to study the vibration of hollow, isotropic, and homogeneous finite length cylinders and frequencies are computed for free-free end boundary conditions in the reference ${ }^{19}$ and compared with the reference. ${ }^{16}$ For solving the aforementioned problem using the graded finite element method developed here, we considered a thick hollow cylinder with freely supported end conditions in which the material distribution is uniform. Therefore, the volume fraction exponent and property coefficients in the 2D-FGM are taken as: $n_{z}=0, n_{r}=0, P_{c 1}=P_{c 2}=P_{m 1}=P_{m 2}=P$, where $P$ is a uniform material properties of the cylinder. Comparison of the results for this case in Table 2 shows good agreement between the two methods again.

A thick hollow cylinder of inner radius $r_{i}=0.5 \mathrm{~m}$, outer radius $r_{o}=1 \mathrm{~m}$ and length $L=2 \mathrm{~m}$ is considered. Effects of volume fraction distribution on the natural frequencies and mode shapes configuration of a simply supported functionally graded thick hollow cylinder are analyzed. The influence of constituent volume fractions is studied by varying the volume fractions of the constituent metals and ceramics. A functionally graded cylinder with two-dimensional gradation of distribution profile has been investigated as well as the case where the axial power law exponent is assumed to be zero, i.e., $n_{z}=$ 0 , and the results of one-dimensional gradation of material distribution can be obtained in the hollow cylinder. The basic materials are as explained in the previous section. Constituent materials are two distinct ceramics and two distinct metals described in Table 1 and Poison's ratio $\nu=0.3$. Volume fractions of materials are distributed according to Eqs. (1a) to (1d). Vibration characteristics of cylinder for some different powers of material composition profile $\mathrm{n} \mathrm{r}$ and $\mathrm{n} \mathrm{z}$ are presented and compared. Dimensionless frequency parameter is assumed as

$$
\Omega=\left(r_{o} \omega\right) \sqrt{\frac{\rho}{G}} .
$$

The longitudinal and radial modes are uncoupled from pure torsional modes when the circumferential wave number is taken as $m=0 .{ }^{19}$ For symmetric boundary conditions, the mode shapes are either symmetric or antisymmetric.

In order to investigate the effect of material distribution profile in the case of non-symmetrical modes, some selected mode shapes were computed for comparison, are shown in following Tables.

Three-dimensional antisymmetric vibrational modes related to non-zero values for circumferential wave numbers $(m \neq 0)$ will be described in the following illustrations. Mode shapes are according to the classification of the modes of thick cylinders used by Wang and Williams. ${ }^{25}$ Axial bending modes, where the circumferential cross section segments bend oppositely in the axial direction, and the radial motion with shearing modes; for this kind the cylinder no longer retains a constant cross sectional along its length. Circumferential in these modes means adjacent segmental elements expand or contract one by one in the circumferential direction. The median circumferential length of an expanding segment becomes longer and the length of the contracting segment becomes shorter. Global modes for these modes is the thick cylinder can be considered to behave as a simple beam vibrating in a transverse direction, a bar vibrating in torsion or as a rod vibrating in a longitudinal direction.

The variations of the mode shapes the circumferential wave numbers $m$ for different values of longitudinal mode numbers for $n_{r}=0.2$ and $n_{z}=0$ is shown in Table 3. Although the displacements of each nodes of the cylinder are calculated, just some cross sections of the cylinder on inner and outer radii are shown due to the nature of the illustration.

The variations of the natural dimensionless frequencies parameter with the circumferential wave numbers, $m$ for different values of radial power exponent while the axial power exponent $n_{z}=0$ is shown in Fig. 2. It is clear that the effect of variation of radial power exponent is more considerable for higher natural frequencies. The same results for $n_{r}=5$ and $n_{z}=0$ is shown in Table 4.

The effect of variation of axial power exponent is considered in Tables 5 and 6 . Mode shapes for two different axial power exponents while the radial power exponent is zero $\left(n_{r}=0\right)$ 


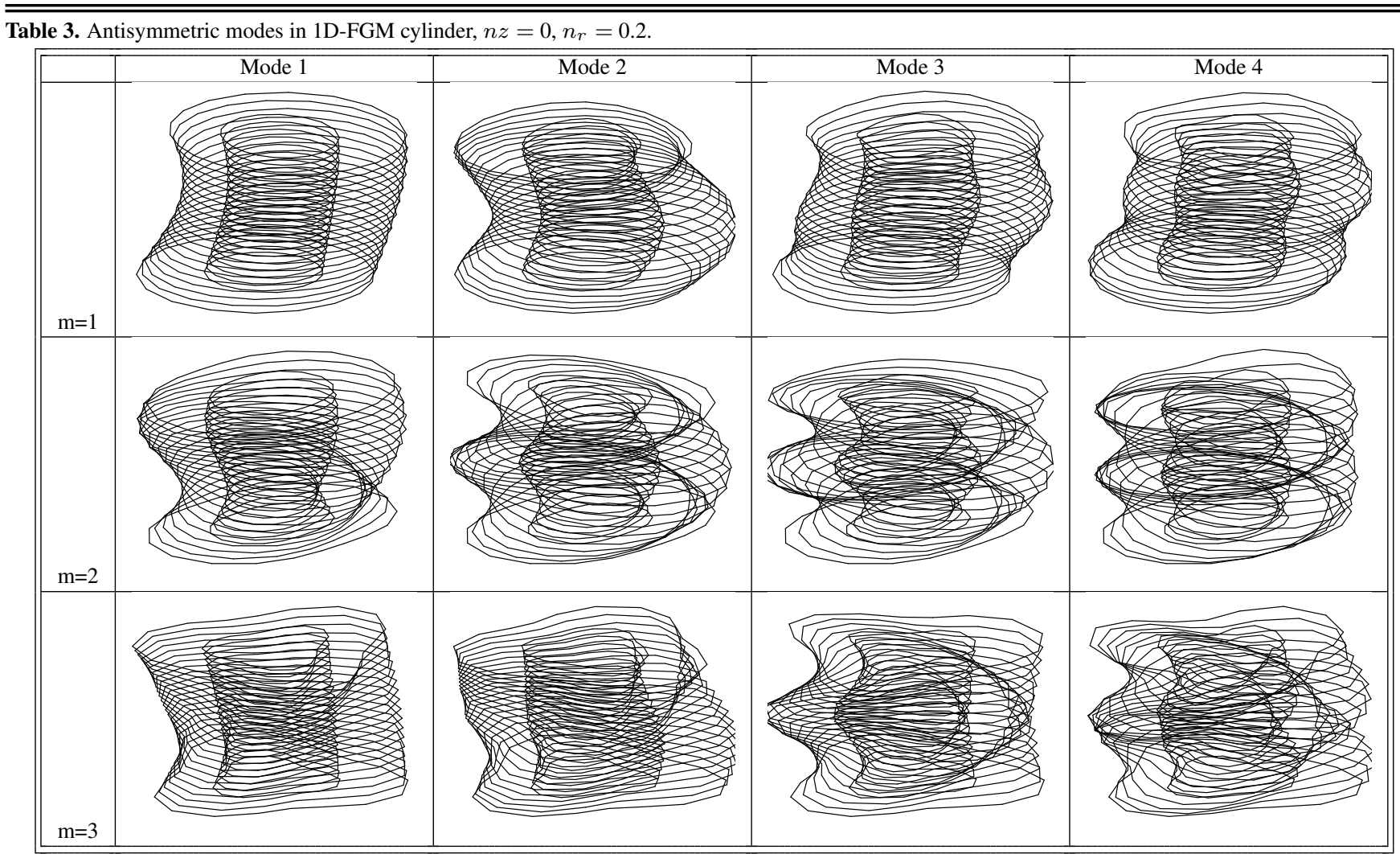

Table 4. Antisymmetric modes in $1 \mathrm{D}-\mathrm{FGM}$ cylinder, $n z=0, n_{r}=5$.

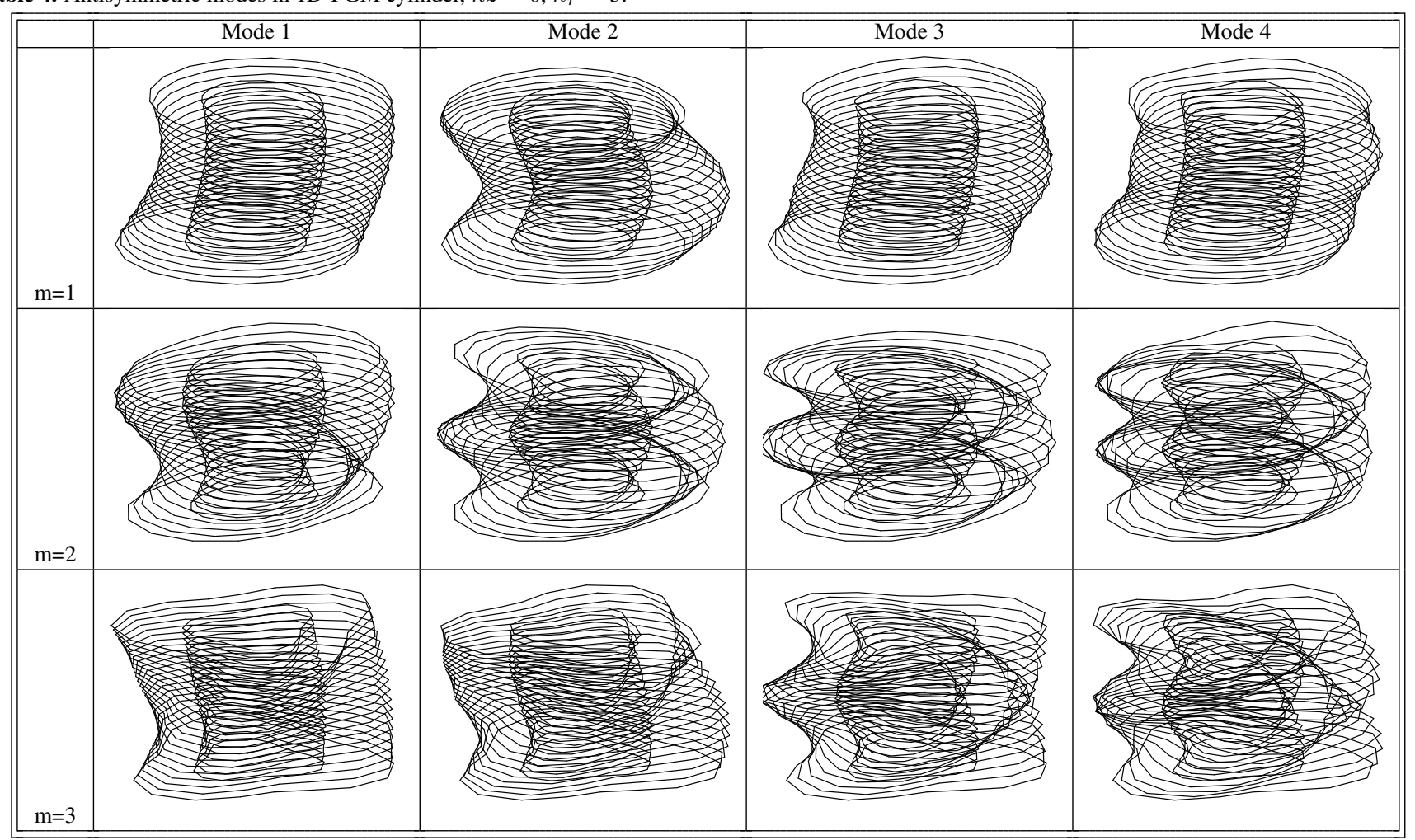

are shown in these tables. The same investigation for natural frequency is also indicated in Fig. 3. It is clear that effect of axial power exponent on the natural frequencies is insignificant when the radial power exponent is zero.

In order to further study the mode configuration due to the power exponent, the 2D-FGM cylinder with both non-zero exponents is considered in Tables 7 and 8.

Variation of natural frequencies with a circumferential wave number for different values of axial power exponent, while the radial power exponent is not zero $\left(n_{r}=2\right)$ is shown in Fig. 4; the influence of the value of $n_{z}$ can be seen here. It is clear that as $n_{z}$ increased, the natural frequencies increased. The natural frequencies and the mode shapes of different kinds of modes vary with the changing material's distribution profile.

It is clear from the results that the natural frequencies and mode shapes, are strongly influenced by the material composition profile. The constituent volume fractions of the constituent materials affect antisymmetric mode shapes particularly in higher mode numbers. It should be noted that although the manufacturing of multidimensional FGM may seem to 
Table 5. Antisymmetric modes in 1D-FGM cylinder, $n_{r}=0, n z=0.2$.

Mode 1

Table 6. Antisymmetric modes in $1 \mathrm{D}-\mathrm{FGM}$ cylinder, $n_{r}=0, n z=5$.

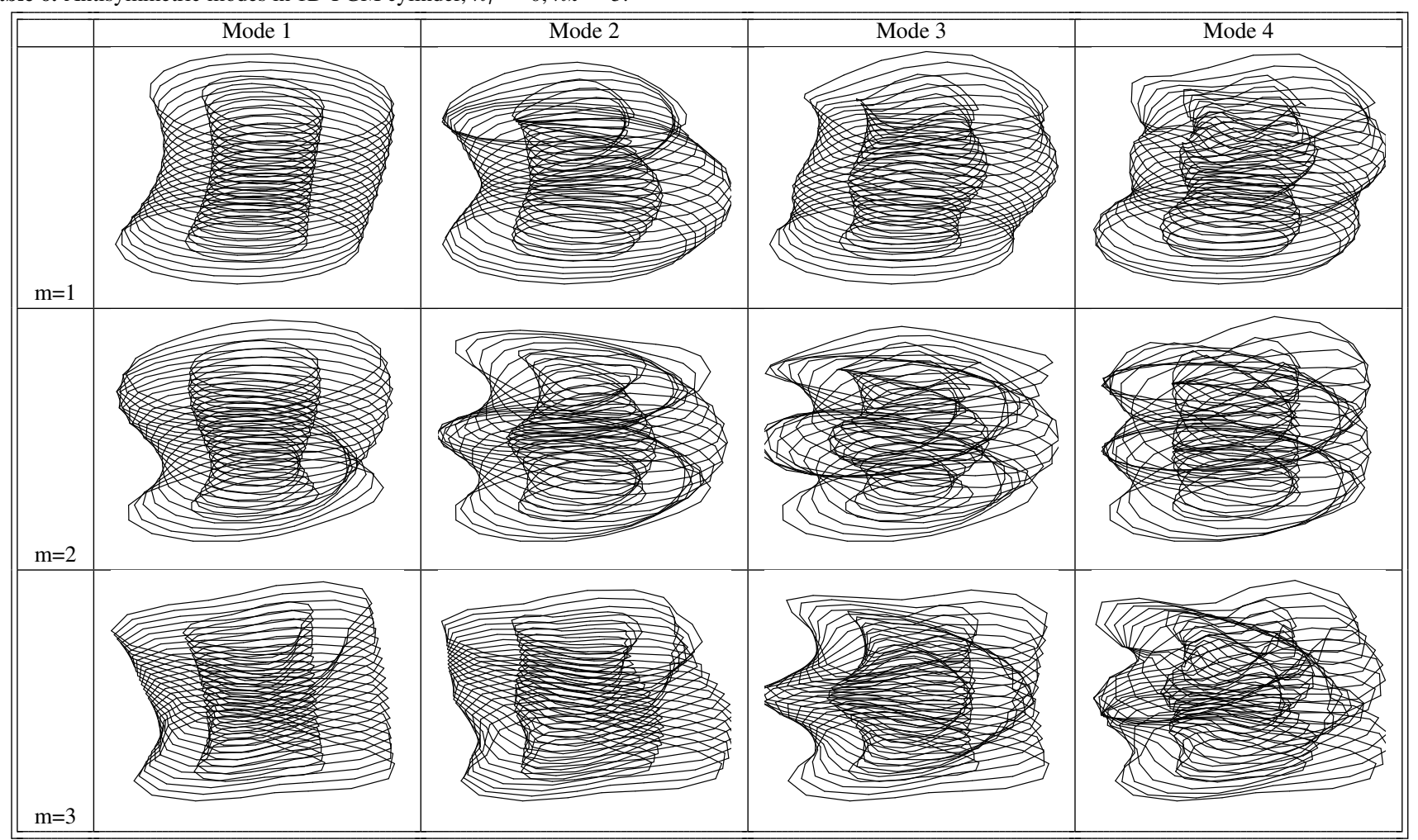

be costly or difficult, these technologies are relatively new, processes such as three-dimensional printing $\left(3 \mathrm{DP}^{\mathrm{TM}}\right)$ and Laser Engineering Net Shaping (LENS ${ }^{\circledR}$ ) can currently produce FGMs with relatively arbitrary tree-dimensional grading. ${ }^{33}$ With further refinement, FGM manufacturing methods may provide designers with more control of the composition profile of functionally graded components with reasonable cost.

\section{CONCLUSIONS}

Based on the three-dimensional mode shapes, a study on the free vibration of simply supported thick hollow cylinder with finite length made of 2D-FGM is presented. Material properties are graded in the thickness and longitudinal directions of the cylinder according to a volume fraction power law distribution. The equations of motion are based on threedimensional elasticity theory and the graded finite element method, which has some advantages to the conventional finite 


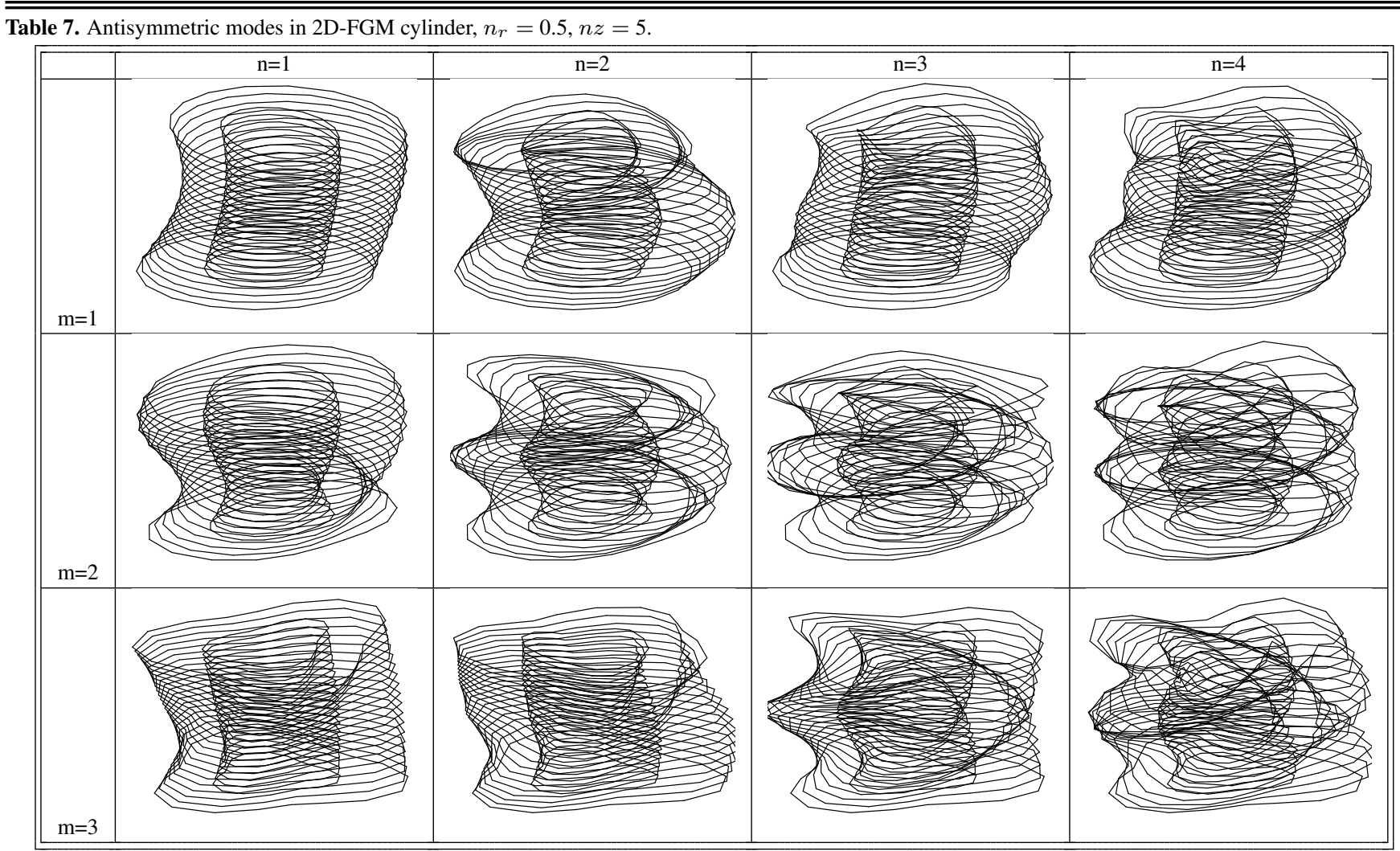

Table 8. Antisymmetric modes in 2D-FGM cylinder, $n_{r}=5, n z=0.5$.

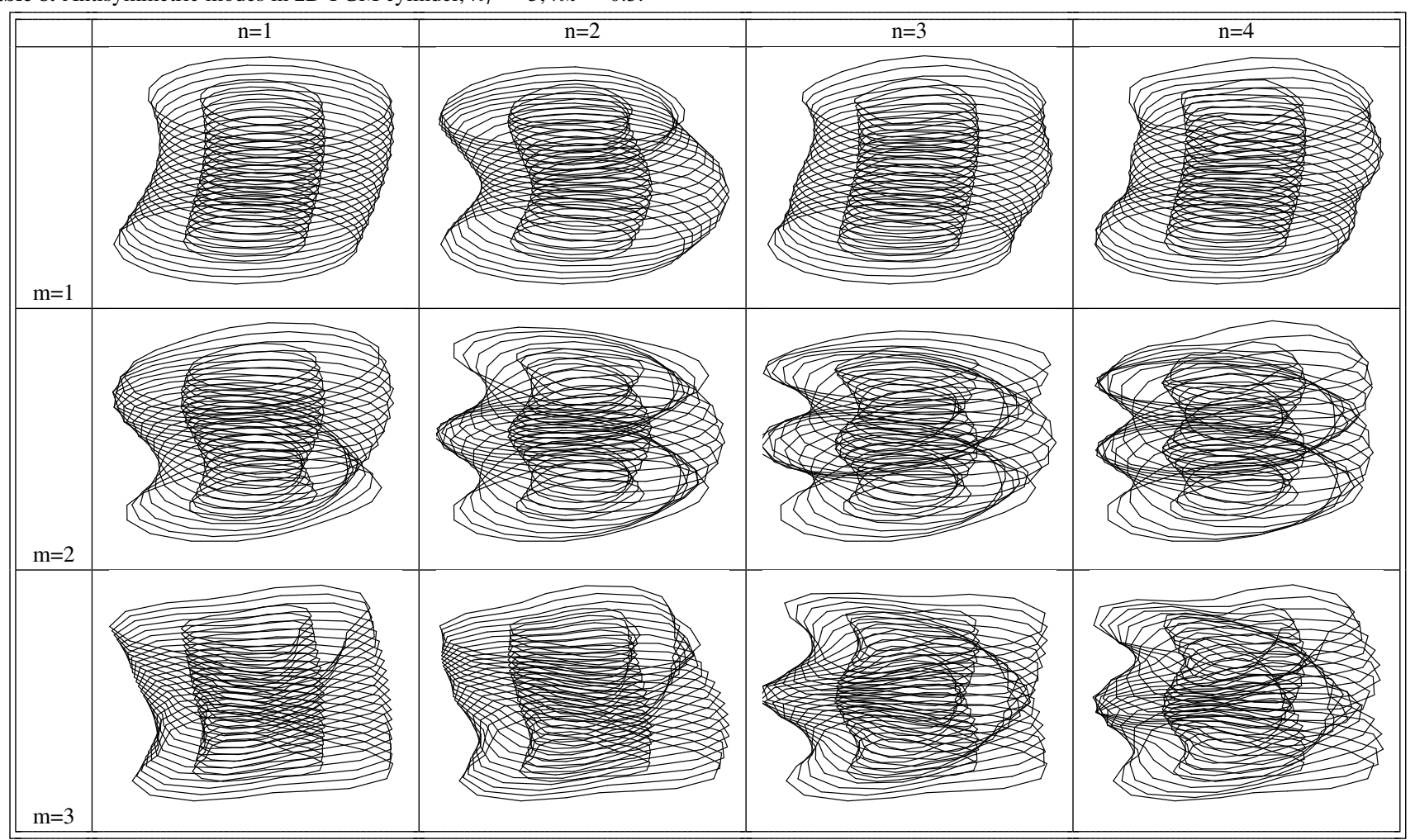

element method and is employed for the solution. The effects of two-dimensional material distribution on the mode shapes characteristics are considered and compared with conventional one-dimensional FGM. Variation of natural frequencies and anti-symmetric three-dimensional mode shapes with circumferential wave number associated with the numerous values of radial and axial power exponents are calculated for 1D- FGM cylinder as well as 2D-FGM cylinder. The study shows that the 2D-FGM cylinder exhibits an interesting frequency and mode shape characteristics when the constituent volume fractions are varied. Based on the achieved results, 2D-FGMs have a powerful potential for designing and optimization of structures under multi-functional requirements.

\section{REFERENCES}

1 Koizumi, M. The concept of FGM Ceramic Transaction, Functionally Graded Materials, 34, 3-10, (1993).

2 Loy, C. T., Lam, K. Y., and Reddy, J. N. Vibra- 


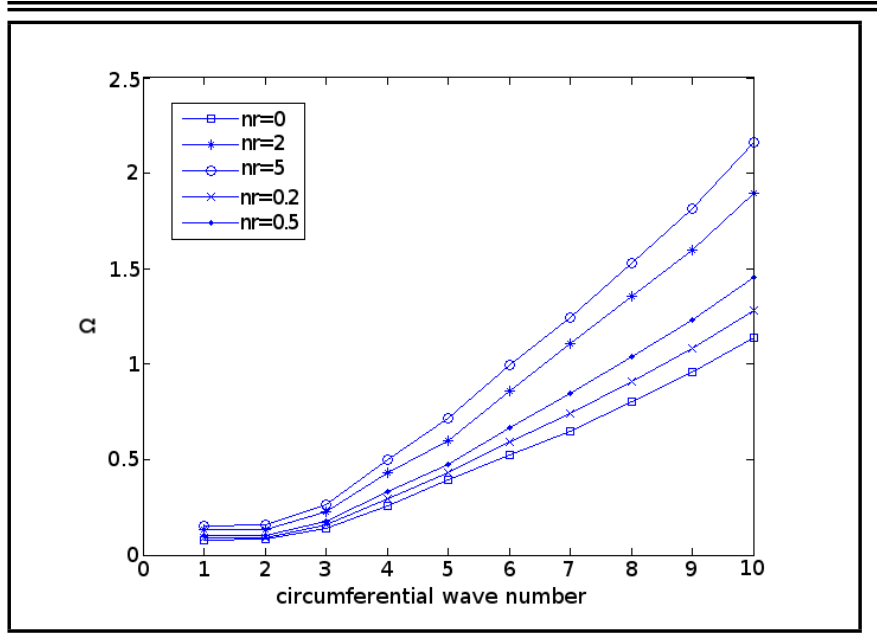

Figure 2. Natural frequencies for various radial power law exponents in 1DFGM cylinder, $n_{z}=0 .{ }^{37}$

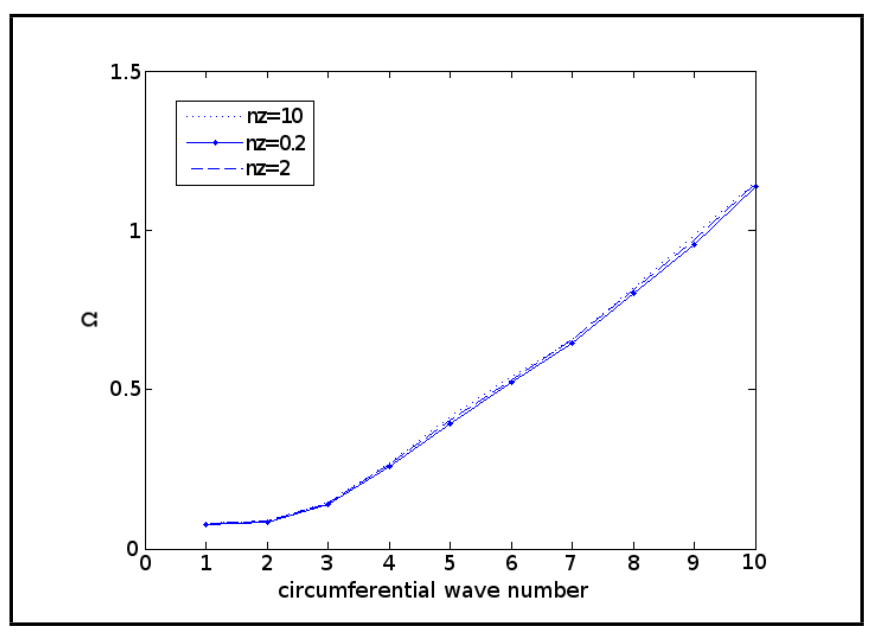

Figure 3. Natural frequencies for various axial power law exponents in 1DFGM cylinder, $n_{r}=0$.

tion of functionally graded cylindrical shells, Int Journal of Mechanical Sciences, 41, 309-324, (1999). https://dx.doi.org/10.1016/s0020-7403(98)00054-x

3 Pradhan, S. C., Loy, C. T., Lam, K. Y., and Reddy, J. N. Vibration characteristics of functionally graded cylindrical shells under various boundary conditions, Applied Acoustics, 61, 111-129, (2000). https://dx.doi.org/10.1016/s0003-682x(99)00063-8

4 Zhao, X., Ng, T. Y., and Liew K. M. Free vibration of two-side simply-supported laminated cylindrical panels via the mesh-free kp-Ritz method, Int Journal of Mechanical Sciences, 46, 123-142, (2004). https://dx.doi.org/10.1016/j.ijmecsci.2004.02.010

5 Patel, B. P., Gupta, S. S., Loknath, M. S., and Kadu, C. P. Free vibration analysis of functionally graded elliptical cylindrical shells using higher-order theory, Composite Structures 69, 259-270, (2005). https://dx.doi.org/10.1016/j.compstruct.2004.07.002

6 Kadoli, R. and Ganesan, N. Buckling and free vibration analysis of functionally graded cylindrical shells subjected to a temperature specified boundary condition, Journal of Sound and Vibration, 289, 450-480, (2006). https://dx.doi.org/10.1016/j.jsv.2005.02.034

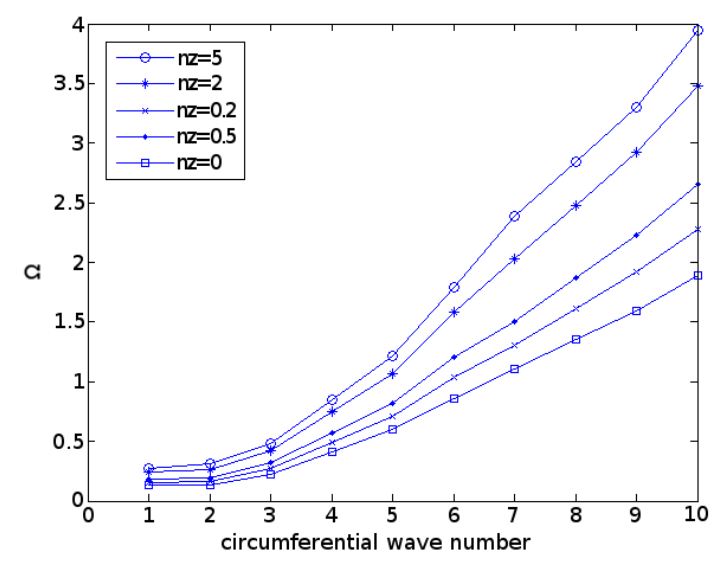

Figure 4. Natural frequencies for various axial power law exponents in 2DFGM cylinder, $n_{r}=2$

7 Chen, W. Q., Bian, Z. G., Lv, C. F., and Ding, H. J. 3D free vibration analysis of a functionally graded piezoelectric hollow cylinder filled with compressible fluid, International Journal of Solids and Structures, 41, 947-964, (2004). https://dx.doi.org/10.1016/j.ijsolstr.2003.09.036

8 Loy, C. T. and Lam, K. Y. Vibration of thick cylindrical shells on the basis of three-dimensional theory of elasticity, Journal of Sound and vibration, 226 (4), 719-737, (1999). https://dx.doi.org/10.1006/jsvi.1999.2310

9 Greenspon, J. E. Flexural vibrations of a thick-walled cylinder according to the exact theory of elasticity, Journal of Aerospace Sciences, 27, 1365-1373, (1957). https://dx.doi.org/10.2514/8.8370

10 Gazis, D. C. Three-dimensional investigation of the propagation of waves in hollow circular cylinders, Journal of the Acoustical Society of America, 31, 568-578, (1959). https://dx.doi.org/10.1121/1.1907754

11 Nelson, R. B., Dong, S. B., and Kalra R. D. Vibrations and waves in laminated orthotropic circular cylinders, Journal of Sound and Vibration, 18, 429-444, (1971). https://dx.doi.org/10.1016/0022-460x(71)90714-0

12 Armenakas, A. E., Gazis, D. S., and Herrmann G. Free vibrations of Circular Cylindrical Shells, Pergamon Press, Oxford, (1969). https://dx.doi.org/10.1016/b978-008-011732-4.50012-x

13 Gladwell, G. and Vijay, D. K. Natural frequencies of free finite-length circular cylinders, Journal of Sound and Vibration, 42 (3), 387-397, (1975). https://dx.doi.org/10.1016/0022-460x(75)90252-7

14 Gladwell, G. and Vijay, D. K. Vibration analysis of axisymmetric resonators, Journal of Sound and Vibration, 42 (2), 137-145, (1975). https://dx.doi.org/10.1016/0022$460 x(75) 90211-4$

15 Hutchinson, J. R. and El-Azhari, S. A. Vibrations of free hollow circular cylinders, Journal of Applied Mechanics, 53, 641-646, (1986). https://dx.doi.org/10.1115/1.3171824

16 So, J. and Leissa, A. W. Free vibrations of thick hollow circular cylinders from three-dimensional analysis, Trans ASME, Journal of Vibrations and Acoustics, 119, 89-95, (1997). https://dx.doi.org/10.1115/1.2889692 
17 So, J. Three dimensional vibration analysis of elastic bodies of revolution, $\mathrm{PhD}$ dissertation, The Ohio State University, Columbus, (1993).

18 Liew, K. M., Hung, K. C., and Lim, M. K. Vibration of stress-free hollow cylinders of arbitrary cross section, Trans ASME, Journal of Applied Mechanics, 62, 718-724, (1995). https://dx.doi.org/10.1115/1.2897005

19 Buchanan, G. R. and Yii, C. B. Y., Effect of symmetrical boundary conditions on the vibration of thick hollow cylinders, Applied Acoustics, 63, 547-566, (2002). https://dx.doi.org/10.1016/s0003-682x(01)00048-2

${ }^{20}$ Cheung, Y. K. and Wu, C. I., Free vibrations of thick, layered cylinders having finite length with various boundary conditions, Journal of Sound and Vibration, 24, 189-200, (1972). https://dx.doi.org/10.1016/0022-460x(72)90948-0

21 Soldatos, K. P. and Hadjigeorgiou, V. P. Three-dimensional solution of the free vibration problem of homogeneous isotropic cylindrical shells and panels, Journal of Sound and Vibration, 137, 369-384, (1990). https://dx.doi.org/10.1016/0022-460x(90)90805-a

22 Jiang, X. Y. 3-D vibration analysis of fiber reinforced composite laminated cylindrical shells, Journal of Vibration and Acoustics, 119, 46-51, (1997). https://dx.doi.org/10.1115/1.2889686

23 Ye, J. Q. and Soldatos, K. P. Three-dimensional vibrations of cross-ply laminated hollow cylinders with clamped edge boundaries, Journal of Vibration and Acoustics, 119, 317323, (1997). https://dx.doi.org/10.1115/1.2889726

24 Singal, R. K. and Williams, K. A theoretical and experimental study of vibrations of thick circular cylindrical shells and rings, Trans ASME, Journal of Vibrations, Acoustics, Stress, and Reliability in Design, 110, 533-537, (1988). https://dx.doi.org/10.1115/1.3269562

25 Wang, H. and Williams, K. Vibrational modes of thick cylinders of finite length, Journal of Sound and Vibration, 191 (5), 955-971, (1996). https://dx.doi.org/10.1006/jsvi.1996.0165

26 Singhal, R. K., Guan, W., and Williams, K. Modal analysis of a thick-walled circular cylinder, Mechanical Systems and Signal Processing, 16 (1), 141-153, (2002). https://dx.doi.org/10.1006/mssp.2001.1420

27 Nemat-Alla, M. Reduction of thermal stresses by developing two dimensional functionally graded materials, Int. J. Solids Structures, 40, 7339-7356, (2003). https://dx.doi.org/10.1016/j.ijsolstr.2003.08.017

28 Dhaliwal, R. S. and Singh, B. M. On the theory of elasticity of a non-homogeneous medium, J Elasticity, 8, 211-219, (1978). https://dx.doi.org/10.1007/bf00052484

29 Clements, D. L. and Budhi, W. S. A boundary element method for the solution of a class of steady-state problems for anisotropic media, Heat Transfer, 121, 462-465, (1999). https://dx.doi.org/10.1115/1.2826000

30 Abudi, J. and Pindera, M. J. Thermoelastic theory for the response of materials functionally graded in two directions, Int. J. Solids Structures, 33, 931-966, (1996). https://dx.doi.org/10.1016/0020-7683(95)00084-4
31 Cho, J. R. and Ha, D.Y. Optimal tailoring of 2D volumefraction distributionsfor heat-resisting functionally graded materials using FDM, Comput Method Appl Mech Eng, 191, 3195-3211, (2002). https://dx.doi.org/10.1016/s00457825(02)00256-6

32 Hedia, H. S., Midany, T. T., Shabara, M. N., and Fouda N. Development of cementless metal-backed acetabular cup prosthesis using functionally graded material, Int J Mech Mater Des, 2, 259-267, (2005). https://dx.doi.org/10.1007/s10999-006-9006-y

33 Goupee, A. J. and Vel, S. Optimization of natural frequencies of bi-directional functionally graded beams, Struct Multidisc Optim, 32 (6), (2006). https://dx.doi.org/10.1007/s00158-006-0022-1

34 Asgari, M., Akhlaghi M., and Hosseini S. M. Dynamic Analysis of Two-Dimensional Functionally Graded Thick Hollow Cylinder with Finite Length under Impact Loading, Acta Mechanica, 208, 163-180, (2009). https://dx.doi.org/10.1007/s00707-008-0133-4

35 Asgari, M. and Akhlaghi M. Thermo-Mechanical Analysis of 2D-FGM Thick Hollow Cylinder using Graded Finite Elements, Advances in Structural Engineering, 14 (6), (2011). https://dx.doi.org/10.1260/1369-4332.14.6.1059

36 Asgari, M. and Akhlaghi, M. Transient thermal stresses in two-dimensional functionally graded thick hollow cylinder with finite length, Arch Appl Mech, 80, 353-376, (2010).

37 Asgari M. and Akhlaghi M. Free Vibration Analysis of 2D-FGM Thick Hollow Cylinder Based on Three Dimensional Elasticity Equations, European Journal of Mechanics A/Solids, 30, 72-81, (2011). https://dx.doi.org/10.1016/j.euromechsol.2010.10.002

38 Santare, M. H., Thamburaj, P., and Gazonas, A. The use of graded finite elements in the study of elastic wave propagation in continuously non-homogeneous materials, Int J Solids Structures, 40, 5621-5634, (2003). https://dx.doi.org/10.1016/s0020-7683(03)00315-9

39 Santare, M. H. and Lambros, J. Use of a graded finite element to model the behavior of non-homogeneous materials, J Appl Mech, 67, 819-822, (2000). https://dx.doi.org/10.1115/1.1328089

40 Kim, J. H. and Paulino, G. H. Isoparametric graded finite elements for nonhomogeneous isotropic and orthotropic materials, J Appl Mech, 69, 502-514, (2002). https://dx.doi.org/10.1115/1.1467094

41 Asgari, M. and Akhlaghi M. Thermo-mechanical Stresses in Two-Dimensional Functionally Graded Thick Hollow Cylinder with Finite Length, $A d$ vances in Structural Engineering, 14 (6), (2012). https://dx.doi.org/10.1177/0021998315622051

42 Boresi, B., Peter, A., and Ken, P. Elasticity in Engineering Mechanics, 2nd ed. Wiley, New York, (1999).

43 Zienkiewicz, O. C. and Taylor, R. L. The Finite element method, VII: Soloid mechanics, 5th edition, ButterworthHeinemann, Oxford, (2000). 